\title{
The Econometric Analysis of Agent-based Models in Finance: An Application
}

\author{
Youwei $\mathrm{Li}^{1}$, Bas Donkers ${ }^{2}$, and Bertrand Melenberg ${ }^{3}$ \\ 1 Queen's University Management School, Queen's University of Belfast, \\ BT7 1NN, Belfast, United Kingdom, y.li@qub.ac.uk \\ 2 Department of Business Economics, Erasmus University Rotterdam, P.O. Box 1738, \\ 3000 DR Rotterdam, The Netherlands, donkers@few.eur.nl \\ 3 Department of Econometrics and O. R., Tilburg University, P.O. Box 90153, \\ 5000 LE Tilburg, The Netherlands, b.melenberg@uvt.nl
}

\begin{abstract}
This paper illustrates how to compare different agent-based models and how to compare an agent-based model with real data. As examples we investigate ARFIMA models, the probability density function, and the spectral density function. We illustrate the methodology in an analysis of the agent-based model developed by Levy, Levy, Solomon (2000), and confront it with the S\&P 500 for a comparison with real life data.
\end{abstract}

\section{Introduction}

Many of the classical models in finance are based upon the assumptions of investor homogeneity and expected utility theory, with as prominent example the Capital Asset Pricing Model (CAPM), see, for example, Cochrane (2001). Although recent research reveals that the assumptions underlying the CAPM are hard to maintain (see Barberis et al. (2001) for a recent discussion), they are still used because of their analytical tractability.

The developments of computational power provide the opportunity to relax these assumptions through the use of simulation techniques. In particular, agent-based models of financial markets have been introduced. These models investigate the interaction of heterogeneous agents, having, for example, different attitudes towards risk or different expectations about future asset returns, and have been studied intensively, see, for example, He and Li (2007a, 2007b), Hommes (2006), LeBaron (2006), Levy et al. (2000), Lux and Marchesi (1999), and references therein. So far, this research has mainly focused on investigating whether a single or some 'representative' simulation runs of an agent-based model shares some important characteristics found in actual financial markets, the so-called stylized facts, such as excess volatility, volatility clustering, or fat tails; see Hommes (2006), for instance. These works provide various ways to explain the stylized facts, but typically focus on different sets of stylized facts. In addition, they match these stylized facts to different degrees. To investigate the relative merits of the various approaches, systematic procedures to investigate 
the differences between two agent-based models or to judge whether an agentbased model is realistic are needed. Such methods have been developed in Li et al. (2006a, 2006b).

The aim of this paper is to illustrative how to use econometric techniques to thoroughly analyze the agent-based models. Although preferably one would like to be able to estimate agent-based models, this is often infeasible. The most important reason is the complexity of the agent-based models, which makes verification of (econometric) identification rather difficult, and thus proving consistency of estimation troublesome. Moreover, in case estimation is possible, the likely heavily nonlinear relationship between observables and unknown parameters to be estimated might seriously complicate estimation. So, only few attempts have been made to estimate agent-based models, see Alfarano et al. (2005) and Boswijk et al. (2007) for examples.

Therefore, in this paper, we constrain our study to the comparison of different agent-based models and on how to compare an agent-based model with actual data. We investigate the comparison of the characteristics that are linked directly to some well-known stylized facts: the ARFIMA parameters, the probability density function, and the spectral density function. Other features of the return process have been investigated in He and Li (2007a, 2007b).

A special feature of agent-based models is that its outcome series can be observed along two dimensions, namely, we can observe the outcome series for each run of the simulation, and we can run the agent-based model independently many times. We shall exploit this possibility heavily in this paper when applying econometric techniques, particularly, when comparing actual data with outcomes of agent-based models.

Throughout this study, we will illustrate the methodology using an early example of the agent-based models: the model developed by Levy et al. (2000) (LLS model from now on). The LLS model applies a standard economic utility maximization approach for the derivation of market activities of individual investors which is augmented by stochastic factors being borrowed from physics. The LLS model is successful in generating very spectacular crashes and upheavals of market prices. Furthermore, varying combinations of time horizons among traders lead to diverse and sometimes surprising results for the time development of the share of wealth owned by different groups of investors. However, a systematic study of the model, such as sensitivity analysis, and a comparison of the model with actual data has never been performed.

The next section briefly introduces the LLS model and its original parameter settings. We then provide a sensitivity analysis and a comparison of two different agent-based models. This is followed by a comparison of the LLS model to real life data, represented by the Standard \& Poor 500 index (hereafter S\&P 500). We end with some concluding remarks. 


\section{The Levy-Levy-Solomon Model}

In the LLS model, Levy et al. (2000), there are two assets: a stock and a bond. The bond is assumed to be a risk free asset, while the stock is a risky asset. The bond is exogenous with infinite supply, so the investors can buy from it as much as they wish at a given rate of return, $r$. The stock is in bounded supply. There are $N$ outstanding shares of the stock. The return on the stock is composed of capital gains and dividend payments. The dividend per share at time t, $D_{t}$, is a random variable that follows a multiplicative random walk: $D_{t}=D_{t-1}(1+z)$, where $z$ is distributed uniformly in the range $\left[z_{1}, z_{2}\right]$. The overall rate of gross return on the stock in period $t, R_{t}$, is then given by $R_{t}=\left(P_{t}+D_{t}\right) / P_{t-1}$ where $P_{t}$ is the stock price at time $t$.

The investors are expected utility maximizers, characterized by the utility index $U(W)=W^{1-\alpha} /(1-\alpha)$, which reflects their personal preference. The investors are divided into two groups, the first group will be referred to as the rational informed investors (RII), and the second group will be referred to as the efficient market believers (EMB).

The RII investors - At time $t$ the RII investors believe that the convergence of the price to the fundamental value will occur in period $t+1$. Furthermore, RII investors estimate the next period fundamental value of stock price $P_{t+1}^{f}$ by $P_{t+1}^{f}=E_{t+1}\left[D_{t+2}\right] /(k-g)$ according to Gordon's dividend stream model. Here, $k$ is the discount factor, and $g$ is the expected growth rate of the dividend, i.e., $g=E(z)$, which is known to the investors. Using $E_{t+1}\left[D_{t+2}\right]=D_{t+1}(1+g)$ and $D_{t+1}=D_{t}(1+z)$, RII investors thus believe that $P_{t+1}=P_{t+1}^{f}$ is given by

$$
P_{t+1}^{f}=D_{t}(1+z)(1+g) /(k-g) .
$$

Investing a proportion $x$ of wealth in the stock at time $t$, the expected utility becomes $E\left\{U\left(W_{t+1}\right)\right\}=E\left\{U\left(W_{t}\left[(1-x) r+x R_{t+1}\right]\right)\right\}$, with $W_{t}$ the wealth at time $t$, and where the expectation is over the rate of return $R_{t+1}=\left(P_{t+1}+\right.$ $\left.D_{t+1}\right) / P_{t}$, with $P_{t+1}=P_{t+1}^{f}$. A solution for this optimization problem can be found by solving the first order conditions.

The EMB investor - EMB investor $i$ has only a limited memory, and uses the most recent $m^{i}$ returns on the stock to estimate the ex ante distribution. At time $t$, each of these past returns on the stock $R_{j,} j=t, t-1, \ldots, t-m^{i}+1$ is given an equal probability $1 / m^{i}$ to reoccur in the next period $(t+1)$. Therefore, the expected utility of EMB investor $i$ is given by

$$
E\left\{U\left(W_{t+1}\right)\right\}=\frac{1}{m^{i}} \sum_{j=1}^{m^{i}} \frac{1}{1-\alpha}\left[W_{t}\left[(1-x) r+x R_{t-j}\right]\right]^{1-\alpha} .
$$

Maximization of this expected utility yields the optimal proportion of wealth, $x^{* i}$, that will be invested in the stock by EMB-investor $i$. To allow for noise around the optimal portfolio choice, LLS assume $x^{i}=x^{* i}+\varepsilon^{i}$ where $\varepsilon^{i}$ is a random variable drawn from a normal distribution with mean zero and standard 
deviation $\sigma$. For simplicity, noise is only added to the portfolio share of stocks for the EMB investors.

Given the stock demand of the RII- and EMB-investors, together with the total supply of $N$ fixed shares, the (temporary) Walrasian equilibrium stock price at time $t, P_{t}$, can be determined. This price leads to updated expectations and a new equilibrium arises in the next period, and so on.

The parameters and initial conditions chosen by Levy et al. (2000) are as follows: $W_{0}=\$ 1000, P_{0}=\$ 20.94, D_{0}=\$ 0.5, r=1 \%, k=4 \%, z_{1}=-7 \%$, $z_{2}=10 \%, \sigma=0.2, \alpha=1.5$. Given quarterly trading period, there are 1,000 investors, with $96 \%$ RII investors and $4 \%$ EMB investors with memory span 5 and 15 . This defines the benchmark economy.

\section{Sensitivity analysis}

In this section we investigate the sensitivity of the benchmark LLS model to changes in initial conditions and parameter values. We illustrate this by estimating the $\operatorname{ARFIMA}(0, d, 0)$-process.

We illustrate the sensitivity analysis in terms of the initial price. The subsequent sensitivity analysis in terms of other initial conditions is performed in a similar way. We first simulate the benchmark model. Next, we keep all the conditions and parameters the same, except for the initial price. Two additional simulations are performed, one with an initial price $P_{0}=26$ that is higher than the benchmark price, and an other one with a lower initial price, namely $P_{0}=16$. Then we look at the impact of these different initial prices on the ARFIMA parameters, and see whether they significantly deviate from those of the benchmark model. Next, we do the same exercises in terms of the initial dividend (with $D_{0} \in\{0.4,0.6\}$ ), the risk aversion parameter (with $\alpha \in\{1.45,1.55\}$ ), the maximal one period dividend decreases (with $z_{1} \in\{-0.08,-0.06\}$ ), and the initial wealth. In the latter case we consider two variations: In the first case (unif.), the initial wealth is uniformly distributed over [500, 1500], while in the second case $(50 \%)$ half of the investors have an initial wealth of 500 and the other half have of 1500. In this study, for each set of parameter, we ran 5,000 independent simulations over 1,000 time periods, and for each run of the model we use the last 152 observations to wash away the initial noise effects, and to match the sample size of the S\&P 500 that we use later on.

Table 1 summarizes the maximum likelihood estimates of the ARFIMA model for the benchmark LLS model, by presenting the average results over the simulations, as well as the numbers of significant parameter estimations. We find on average a negative value of $d$, which is significant in only $7.6 \%$ out of the 5,000 simulations. So, based on a single simulation, we would accept most of the times the hypothesis $d=0$ of no fractional integration. However, combining the 5,000 simulations, we find a $t$-value of $t=-23.80$, so that we clearly have to reject the hypothesis $d=0$ ! As could be expected, the use of more simulations can thus substantially increase the power of the tests that are performed. 
Table 1. Estimates of $\operatorname{ARFIMA}(0, d, 0)$ for the benchmark economy

\begin{tabular}{cccccc}
\hline & Coefficient & Std. & $p$-value & $95 \%$ CI & Sig\% \\
\hline$(0, d, 0)$ & -0.0272 & 0.0808 & $0.5210[-0.0294,-0.0250]$ & 7.6 \\
\hline
\end{tabular}

Note: The numbers under Coefficients, Std., and $p$-value are averages over 5,000 simulations, 'Sig\%' reports the percentage of the estimates that are significant at $5 \%$ level.

For different initial conditions and parameter settings, we report the estimates of the ARFIMA $(0, d, 0)$ model in Table 2 . In Table 3 we report the $t$-test for the difference of estimated $d$ between the benchmark model and the models with different initial parameters. We see from Table 3 that the LLS model is rather insensitive with respect to the initial prices, initial dividend, and risk aversion parameter in terms of $d$. However, the changes of maximal one-period dividend decrease $z_{1}$ has a big impact. The reason seems to be that the dividend process is the driving force in the LLS model, and a change in $z_{1}$ changes the whole distribution of the dividend process. When half of the investors is endowed with $\$ 500$ and the other half endowed with $\$ 1500$, the difference in $d$ is not significant. However, the difference in $d$ is significant when all of the investors initial wealth is drawn from a uniform distribution on $[500,1500]$.

Table 2. Estimates of $\operatorname{ARFIMA}(0, d, 0)$ model

\begin{tabular}{ccccccc}
\hline & & $d$ & Std. & $p$-value & $95 \%$ CI & Sig\% \\
\hline \multirow{2}{*}{$P_{0}$} & 16 & -0.0286 & 0.0807 & 0.5201 & {$[-0.0308,-0.0264]$} & 7.7 \\
& 26 & -0.0282 & 0.0807 & 0.5247 & {$[-0.0304,-0.0260]$} & 7.6 \\
\hline \multirow{2}{*}{$D_{0}$} & 0.4 & -0.0272 & 0.0808 & 0.5228 & {$[-0.0294,-0.0250]$} & 7.4 \\
& 0.6 & -0.0247 & 0.0807 & 0.5193 & {$[-0.0269,-0.0225]$} & 6.7 \\
\hline \multirow{2}{*}{$\alpha$} & 1.45 & -0.0281 & 0.0808 & 0.5172 & {$[-0.0303,-0.0259]$} & 7.9 \\
& 1.55 & -0.0273 & 0.0808 & 0.5233 & {$[-0.0295,-0.0251]$} & 7.4 \\
\hline \multirow{2}{*}{$z_{1}$} & -0.08 & 0.0077 & 0.0837 & 0.6621 & {$[0.0063,0.0091]$} & 0.7 \\
& -0.06 & -0.1806 & 0.0707 & 0.0848 & {$[-0.1827,-0.1785]$} & 70 \\
\hline \multirow{2}{*}{$W_{0}$} & unif. & -0.0239 & 0.0809 & 0.5241 & {$[-0.0261,-0.0217]$} & 6.9 \\
& $50 \%$ & -0.0279 & 0.0808 & 0.5230 & {$[-0.0301,-0.0257]$} & 7.7 \\
\hline
\end{tabular}

Table 3. The $t$-test for the sensitivity analysis in terms of $\operatorname{ARFIMA}(0, d, 0)$ estimates

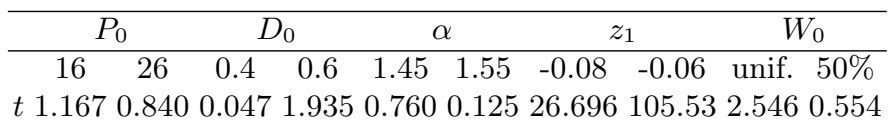


A sensitivity analysis ${ }^{4}$, like the one above, may be of help in determining which parameters or initial conditions, in particular, to use when one would like to calibrate (or estimate) an agent-based model using actual data. In case of the LLS model, the dividend process seems to be an appropriate choice in a calibration exercise. By modeling it flexibly, one might become able to describe a wide range of potential distribution characteristics, so that a calibration exercise might become successful. For estimation purposes, the sensitivity analysis might shed some light on (econometric) identifiability of the parameters, with parameters having no effect on the return process not, or at most hardly, being identified.

\section{Comparing two LLS models}

Traditional theory, such as efficient market theory, casts doubts on profitability of technical trading strategies. It is interesting to study the performance of different groups of traders in the LLS setting. Zschischang and Lux (2001) investigate what will happen in the LLS model when a new type of investors is introduced, namely the constant portfolio investors, who always invest a constant proportion of their wealth in the stocks. Zschischang and Lux (2001) investigate the LLS model where initially all the investors are EMB investors (consisting of three or more subgroups). The authors found, when the market is invaded by only a small amount of constant portfolio investors (1\%), that, even when these new investors are endowed with a small initial wealth and hold $1.5 \%$ of their portfolio in the stock, they eventually achieve dominance and asymptotically gain $100 \%$ of the available wealth. Here we consider a model where $0.5 \%$ of the investors are constant portfolio investors instead of RII-investors (having the same initial wealth as the other investors). These constant portfolio investors invest $1.5 \%$ of their wealth in the stock. We keep the other characteristics the same as in the benchmark model.

We performed a Wald test to investigate whether the introduction of the constant portfolio investors has a significant impact. Comparisons with the benchmark model are made in terms of log return, log price, and proportion of total wealth held by the two groups of EMB investors with different memory span. The wealth distribution is important as the influence of each type of investor depends strongly on the wealth they possess.

We consider two cases to investigate possible differences of these quantities over time. In CaseI, the periods under consideration are the last 100 periods, i.e., $t=901,902, \ldots, 1000$; in CaseII , only six time points are considered, $t=$ $500,600, \ldots, 900,1000$. The resulting test statistics are summarized in Table 4. In the column labeled 'Log-return', the first row reports the Wald statistics of the benchmark economy; for instance, 101.88 is the Wald statistic corresponding to the null hypothesis of equality of the average log return in the benchmark

\footnotetext{
4 The sensitivity analysis in terms of the ARFIMA $(1, d, 1)$, the probability density function, and the spectral density function yields the results more or less in line with the $\operatorname{ARFIMA}(0, d, 0)$-case (not reported, see Li et al. 2006a, 2006b).
} 
economy in periods $t=901,902, \ldots, 1000$ (with degrees of freedom between brackets), the second row reports the Wald statistics for equality of the logreturns in the new economy, and the third row reports the results of comparing the new economy with the benchmark model. To indicate the groups of EMB investors with different memory spans, we abbreviate memory span to $\mathrm{ms}$. It is clear that none of the comparison statistics is significant, thus, the constant portfolio investors do not cause a significant impact on the economy. We do see substantial differences across time in both economies for the price level.

Table 4. The comparison results with the benchmark model in terms of the mean

\begin{tabular}{ccccc}
\hline & Log-return & Log-price & Wealth $(m s=5)$ & Wealth $(m s=15)$ \\
\hline \multirow{3}{*}{ CaseI } & $101.88(99)$ & $42085.5(99)$ & $122.20(99)$ & $125.10(99)$ \\
& $101.68(99)$ & $43413.2(99)$ & $124.84(99)$ & $113.73(99)$ \\
& $77.95(100)$ & $76.95(100)$ & $97.29(100)$ & $79.13(100)$ \\
\hline \multirow{3}{*}{ CaseII } & $9.53(5)$ & $192436.7(5)$ & $383.93(5)$ & $772.40(5)$ \\
& $2.65(5)$ & $194884.1(5)$ & $388.87(5)$ & $757.85(5)$ \\
& $8.33(6)$ & $7.44(6)$ & $3.37(6)$ & $2.45(6)$ \\
\hline
\end{tabular}

Figure 1 explains why. The wealth of the constant portfolio investors decreases gradually. In the Zschischang and Lux-analysis the constant portfolio investors are the only investors who are at the opposite side of the market in case of the cycles, so that eventually they are able to gain all wealth. But in the model considered here, the RII investors for a large part take over this role by buying or selling, depending on the price being lower or higher than its fundamental value, resulting in a gradually decreasing wealth held by the constant portfolio investors.

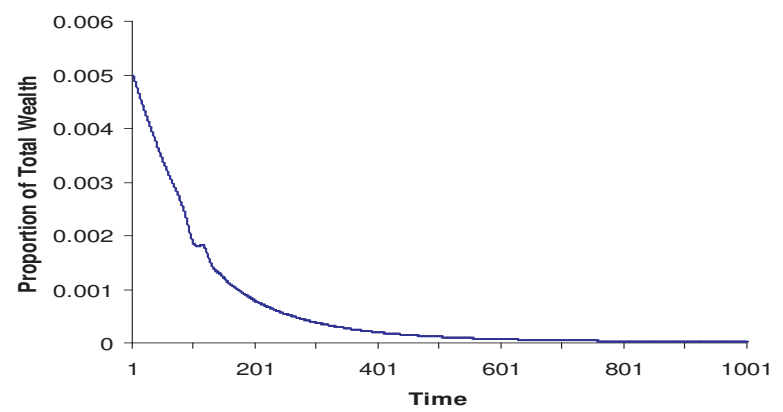

Fig. 1. Wealth held by constant portfolio investors, averaged over 5,000 simulations. 


\section{Comparing the LLS model with real life data}

From Levy et al. (2000), it seems that the LLS model is able to generate realistic price behaviour at a quarterly frequency. So, we use quarterly data of the S\&P 500 from Datastream as representation of the real life situation, which runs from the first quarter of 1965 to the first quarter of 2003. We compare the LLS model with the real world in terms the coefficients of the $\operatorname{ARFIMA}(0, d, 0)$ process, the probability density function, and the spectral density function. First, we estimate the $\operatorname{ARFIMA}(0, d, 0)$ model for the S\&P 500 and the results are reported in Table 5 . The parameter $d$ is not significant, so there is no evidence of long memory in the quarterly stock return process. More important for our analysis is that the estimated $d$ from the LLS model (the average reported in Table 1) lies within the $95 \%$ confidence interval of estimates of $d$ from actual data, which is $(-0.1506,0.114)$.

Table 5. Estimates of $\operatorname{ARFIMA}(0, d, 0)$ for the $\mathrm{S} \& \mathrm{P} 500$

\begin{tabular}{lcccc}
\hline & Coefficient & Std. & $t$-value & $p$-value \\
\hline$(0, d, 0)$ & -0.0183 & 0.0675 & -0.272 & 0.786 \\
\hline
\end{tabular}

Second, we consider the marginal probability density function, which can be estimated non-parametrically (see, for example, Pagan and Ullah, 1999). We apply the bootstrap to construct confidence intervals, where we follow the procedure suggested by Hall (1992). Obviously, a set of $(1-\alpha)$ point-wise confidence intervals constructed for a discretized finite interval will not achieve a $(1-\alpha)$ joint coverage probability. So, we also consider a uniform confidence band, following the suggestions by Hall (1993), see Li et al. (2006b) for more details.

We plot the probability density functions of the LLS model, the S\&P 500, and that of a normal distribution with mean and variance equal to that of the S\&P 500 in Figure 2. In addition, we also plot a 95\% confidence interval in Figure 2(a) and a 95\% uniform confidence band in Figure 2(b) around the S\&P 500 estimates. The normal density fits within both the point-wise and uniform confidence intervals, but the LLS model based average density does not fall within the point-wise confidence interval in its entirety, while it almost fits within the uniform confidence band. Thus, the actual return distribution as a whole can be reproduced by the LLS model according to the confidence band, but the distribution seems to be shifted somewhat to the right, relative to the S\&P 500 distribution.

Next, we consider the spectral density function, which is a standard way of investigating the dynamics commonly used in the economics literature (see, for instance, Diebold et al., 1998). The spectral analysis yields a complete secondorder comparison of the dynamic properties of model and data, providing a complete summary of the time series dynamics. We construct confidence intervals based on the procedure of bootstrapping kernel estimation proposed by Franke 

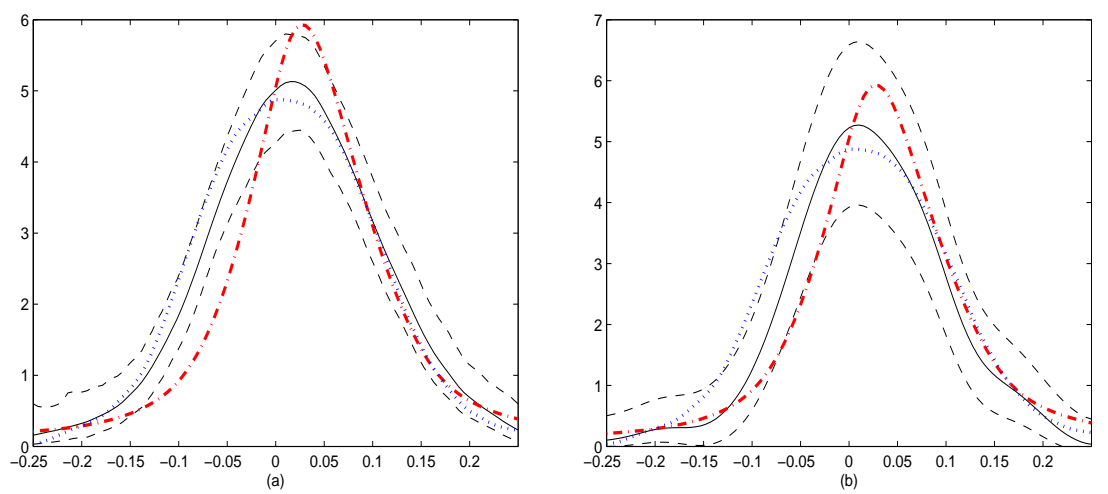

Fig. 2. The density functions of the LLS model (dash-dot line), normal distribution (dot line), and of the S\&P 500 with confidence intervals (a) and confidence band (b)

and Härdle (1992). We construct a uniform confidence band similar to that of Swanepoel and van Wyk (1986) but with parametric estimation, see Li et al. (2006b) for more details.

We present the estimated spectral density function for the S\&P 500, and its corresponding 95\% confidence intervals in Figure 3. In Figure 3(a) we plot the averaged spectral density function over 5,000 simulations, and in Figure 3(b) we do the same for the uniform confidence band. We find that, except for a few rather small frequencies, the LLS based spectral densities lies outside of the $95 \%$ confidence bands of the spectral density function of the S\&P 500. When we compare the frequencies corresponding to the peaks of the spectra, which describe the cycles that dominate the cyclical behaviour of the dynamics, we see that they are not at the same pace. Thus, there seems to be a large difference when we assess the second order moments between the LLS generated data and the real life data. The distribution of memory spans in the economy, however, can be expected to strongly affect the cyclical patterns in the return process and the resulting spectral density. The differences displayed in Figure 3 do suggest that substantial changes are required. We do note that matching the complete spectral density will be far from trivial for many models.

\section{Conclusion}

Econometric techniques can be used to study the agent-based models more thoroughly than the traditional eye-balling of the results, and we argue that this should be done more often. We exploit a unique feature, our control over the number of simulations, which creates one additional dimension for the asymptotic properties of the test statistics. This allows us to ignore the estimation uncertainty present in the simulations when we compare the agent-based models with real life data. Hence, we only need to account for the estimation uncertainty 

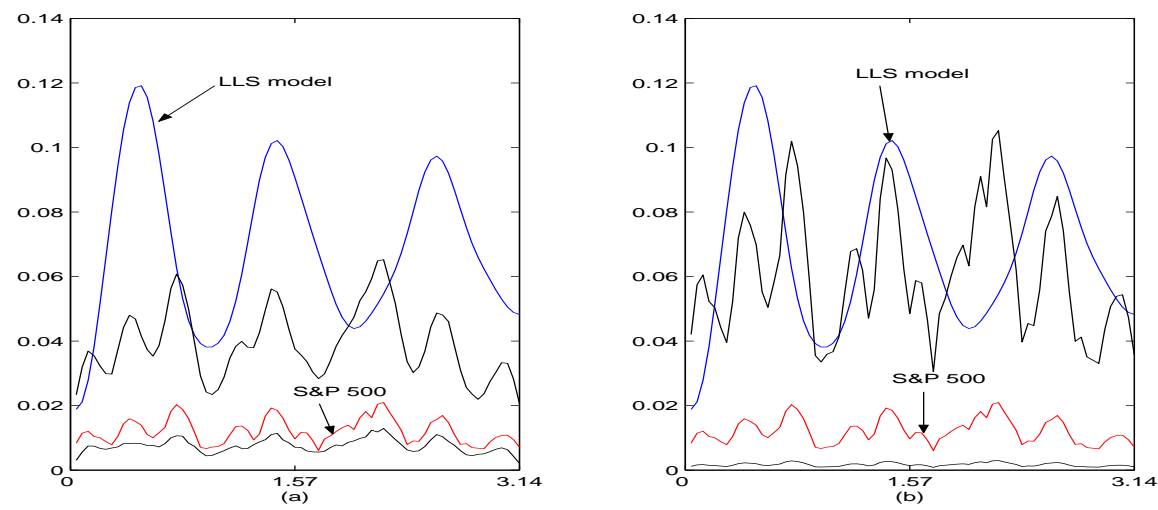

Fig. 3. The spectral density functions with its $95 \%$ confidence intervals (a) and uniform confidence band (b) of the S\&P 500 and the LLS model

of the features of the actual data. In addition, when comparing agent-based models, the control over the number of simulations provides control over the power of the tests. As an example, only $7.6 \%$ of the simulation runs resulted in a significant degree of fractional integration, while there was clear evidence of fractional integration based on all the simulation runs simultaneously.

We illustrate the use of the statistical and econometric techniques by studying one of the earlier agent-based models, the LLS model. The results from our sensitivity analysis indicate that the LLS model is robust with respect to the changes in the initial prices, the initial dividend, the risk aversion parameter, and the initial wealth. However, a change in the one period maximal decrease of dividend has a serious impact, likely, because this changes the whole distribution of the dividend process, and the dividend process is the driving force in the LLS model. This analysis also highlights the problems of estimating agent-based models, as many parameters might not affect the eventual return process and hence will not be identified.

We also illustrate how to compare the LLS generated data with the actual data. We find that while the LLS model seems to be able to provide a good description when the actual data are described by means of an $\operatorname{ARFIMA}(0, d, 0)$ model, the LLS model is not fully in line with real data in terms of other characteristics. Taking into account the sensitivity to the dividend process, a natural way to proceed seems to be to calibrate the LLS model, focussing, in particular, on a parametrization of the dividend process.

\section{References}

Alfarano, S., Lux, T. and Wagner, F.: Estimation of agent-based models: The case of an asymmetric herding model. Computational Economics 26 (2005) 19-49

Barberis, N., Huang, M. and Santos, T.: Prospect theory and asset prices. Quarterly Journal of Economics 116 (2001) 1-54 
Boswijk, H.P., Hommes, C. and Manzan, S.: Behavioral heterogeneity in stock prices. Journal of Economic Dynamics and Control 31 (2007) 1938-1970

Cochrane, J.H.: Asset pricing. Princeton University Press, Princeton (2001)

Diebold, F. X., Ohanian, L., Berkwitz J.: Dynamic equilibrium economies: A framework for comparing models and data. Review of Economic Studies 65 (1998) 433-452

Franke, J., Härdle, W.: On bootstrapping kernel spectral estimates. The Annals of Statistics 20 (1992) 121-145

Hall, P.: Effect of bias estimation on converage accuracy of bootstrap confidence intervals for a probability density. The Annals of Statistics 20 (1992) 675-694

Hall, P.: On Edgeworth expansion and bootstrap confidence bands in nonparametric curve estimation. J. R. Statist. Soc. B 55 (1993) 291-304

He, X., Li, Y.: Heterogeneity, convergence, and autocorrelations. Quantitative Finance (2007b) (to appear)

He, X., Li, Y.: Power-law behaviour, heterogeneity, and trend chasing. Journal of Economic Dynamics and Control 31 (2007b) 3396-3426

Hommes, C.: Heterogeneous agent models in economics and finance. Handbook of Computational Economics, Volume 2, Edited by K.L. Judd and L. Tesfatsion, Elsevier Science (2006)

LeBaron, B.: Agent-based computational finance. Handbook of Computational Economics, Volume 2, Edited by K.L. Judd and L. Tesfatsion, Elsevier Science (2006)

Levy, M., Levy, H., Solomon, S.: Microscopic simulation of financial markets. Academic Press New York (2000)

Li, Y., Donkers, B., Melenberg, B.: Econometric analysis of microscopic simulation models. Tilburg University, CentER Discussion Papers 2006-99 (2006a). Available at: $\langle$ http : //ssrn.com/abstract $=939518\rangle$

Li, Y., Donkers, B., Melenberg, B.: The nonparametric and semiparametric analysis of microscopic simulation models. Tilburg University, CentER Discussion Papers 2006-95 (2006b). Available at: 〈http ://ssrn.com/abstract $=939510\rangle$

Lux, T., Marchesi M.: Scaling and criticality in a stochastic multi-agent model of financial markets, Nature, 397 (1999) 498-500

Pagan, A., Ullah, A.: Nonparametric econometrics. Cambridge University Press (1999)

Swanepoel J. W. H., van Wyk, J. W. J.: The bootstrap applied to power spectral density function estimation. Biometrika 73 (1986) 135-141

Zschischang, E., Lux, T.: Some new results on the Levy, Levy and Solomon microscopic stock market model. Physica A 291 (2001) 563-573 